\title{
REDUCTION OF THE DISPERSION ERROR IN THE INTERPOLATED DIGITAL WAVEGUIDE MESH USING FREQUENCY WARPING
}

\author{
Lauri Savioja and Vesa Välimäki \\ Laboratory of Acoustics and Audio Signal Processing, Helsinki University of Technology, \\ P.O. Box 3000, FIN-02015 HUT, Espoo, Finland \\ E-mail: Lauri.Savioja@hut.fi, Vesa.Valimaki@hut.fi \\ http://www.acoustics.hut.fi/
}

\begin{abstract}
The digital waveguide mesh is an extension of the one-dimensional digital waveguide technique. The mesh is used for simulation of two- and three-dimensional wave propagation in musical instruments and acoustic spaces. The rectangular digital waveguide mesh algorithm suffers from direction-dependent dispersion. By using the interpolated mesh, nearly uniform wave propagation characteristics are obtained in all directions. In this paper we show how the dispersion error of the interpolated mesh can be reduced by frequency warping. By using this technique the bandwidth where the frequency accuracy is within $1 \%$ tolerance is more than doubled.
\end{abstract}

\section{INTRODUCTION}

One-dimensional digital waveguides are a discrete numerical method used to model musical instruments, such as string and wind instruments [1,2]. Two-dimensional (2-D) and three-dimensional (3-D) extensions of digital waveguides have been proposed for simulation of plates, drums [3, 4], and acoustic spaces [5]. Another method which is similar can be obtained by using multidimensional wave digital filters [6].

In the original multi-dimensional digital waveguide mesh, the wave propagation speed is a function of propagation direction [3, $4,7]$. By using more advanced structures, such as the triangular mesh $[8,9,10]$, nearly uniform wave propagation characteristics can be obtained in all directions. Another way how this can be achieved is by using an interpolation technique with the rectangular mesh as presented in an earlier study [7]. Although the dispersion error of the triangular mesh is very small, the rectangular structure is still attractive for some applications. It is conceptually simple: the indexing of the mesh nodes is easy, and the tesselation of a given plane or space is straightforward, especially in the case of rectangular objects.

Also in the interpolated rectangular mesh there still remains dispersion error which increases with frequency [7]. The error is not very harmful in music synthesis applications, but in highaccuracy numerical simulations the dispersion limits the valid bandwidth of simulations. In this paper we show that the dispersion error in the interpolated rectangular digital waveguide mesh can be reduced by frequency warping. This is possible because the error is nearly independent of propagation direction. We examine the 2-D case, but the results may be extended to three dimensions.

The interpolated rectangular digital waveguide mesh is briefly described and analyzed in Section 2. In Section 3, the frequencywarping technique is introduced. The performance of the proposed method is illustrated in Section 4 with simulated examples. Section 5 concludes the paper.

\section{INTERPOLATED MULTI-DIMENSIONAL DIGITAL WAVEGUIDE MESH}

A multi-dimensional rectangular digital waveguide mesh is a regular array of 1-D digital waveguides arranged along each perpendicular dimension, interconnected between all the unit delay elements. A difference equation can be derived for the nodes of an $N$-dimensional rectangular mesh [3, 4]:

$$
p_{c}(n)=\frac{1}{N} \sum_{l=1}^{2 N} p_{l}(n-1)-p_{c}(n-2)
$$

where $p$ represents the displacement at a junction at time step $n$, subscript $c$ denotes the junction to be calculated, and index $l$ represents its $2 N$ axial neighbors.

Ideally, waves should propagate at the same speed in all directions. In the original digital waveguide mesh, however, sample updates occur along the $2 N$ axial directions only. This approximation causes inaccuracies in the wave propagation speed [3, 4, 7]. The ratio between the speed in the original 2-D digital waveguide mesh and the ideal speed is presented by the dispersion factor $[10,4]$ :

$$
k(\xi)=\frac{\sqrt{2}}{2 \pi \xi} \arctan \frac{\sqrt{4-b^{2}}}{b}
$$

where $\xi_{1}$ and $\xi_{2}$ are the normalized spatial frequency coordinates, $\xi=\sqrt{\xi_{1}^{2}+\xi_{2}^{2}}$ and $b=\cos \left(2 \pi \xi_{1} c T\right)+\cos \left(2 \pi \xi_{2} c T\right)$, and $c$ is the ideal wave propagation speed, that is, $c=\frac{1}{\sqrt{2}}$ spatial samples per sampling interval $T$. The relative frequency error (RFE) is obtained from the dispersion factor as

$$
R F E(f)=\frac{k(\xi)-k_{D C}}{k_{D C}} \cdot 100 \%
$$

where $k_{D C}=\lim _{\xi \rightarrow 0} k(\xi)$ and $f$ is the normalized temporal frequency such that $f=c \xi$. The relative frequency error in axial and diagonal directions is shown in Fig. 1(a). There is no error in the diagonal direction but in the axial direction, where the maximal error occurs, the wave propagation speed decreases with frequency so that $1 \%$ error is reached at $0.077 f_{s}$, where $f_{s}$ is the sampling frequency. Note that we show the RFE as a function of temporal frequency, since we want to compare our results against ideal results that are also available as a function of $f$ (see Section 4). 

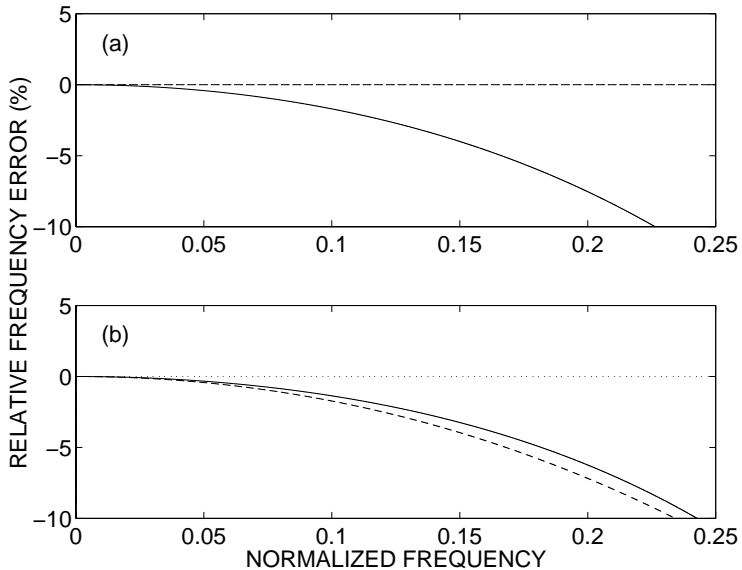

Figure 1: Relative frequency error in axial (solid) and diagonal (dashed) directions in (a) the original, and (b) the interpolated digital waveguide mesh, as a function of normalized temporal frequency, where 0.5 corresponds to the Nyquist frequency. The dotted line indicates the zero error. Note that there is no error in the original mesh in diagonal direction and thus the dashed and dotted lines overlap in (a).

However, in previous literature the error has been presented as a function of spatial frequency $[3,4,8,9,10]$.

In the 2-D digital waveguide mesh algorithm each node in the mesh has four neighbors. To obtain more homogeneous wave propagation characteristics we insert a unit delay element also between diagonal neighbors. Since those lines end between actual mesh nodes they must be interpolated [11]. We have used bilinear interpolation resulting in the following equation [7]:

$$
p_{c}(n)=\frac{1}{4} \sum_{l=1}^{3} \sum_{k=1}^{3} h_{l, k} p_{l, k}(n-1)-p_{c}(n-2)
$$

where $p_{l, k}$ represent displacements at $p_{c}$ and its eight neighboring nodes and $h_{l, k}$ are the weighting coefficients of each node such that $h_{d}=h_{11}=h_{13}=h_{31}=h_{33}=1 / 2, h_{a}=h_{12}=$ $h_{21}=h_{32}=h_{23}=\sqrt{2}$, and $h_{c}=h_{22}=6-4 \sqrt{2}$. In this structure there is no magnitude dissipation error [7]. The theoretical wave propagation speed in the interpolated mesh can be determined from (2) with

$$
\begin{aligned}
b & =\frac{1}{2}\left\{h_{a}\left[\cos \left(2 \pi \xi_{1} c T\right)+\cos \left(2 \pi \xi_{2} c T\right)\right]\right. \\
& \left.+h_{d}\left[\cos \left(2 \pi \delta_{+} c T\right)+\cos \left(2 \pi \delta_{-} c T\right)\right]+\frac{h_{c}}{2}\right\}
\end{aligned}
$$

where $\delta_{+}=\xi_{1}+\xi_{2}$ and $\delta_{-}=\xi_{1}-\xi_{2}$. Figure 1(b) shows the RFE in the interpolated digital waveguide mesh in axial and diagonal directions which correspond to the minimal and maximal error, respectively. There is still dispersion, but now it is nearly independent of direction.

The interpolation technique can be also applied to the 3-D rectangular digital waveguide mesh as described in [12]. The results show that also in that case it is possible to obtain nearly uniform wave propagation characteristics in all directions.

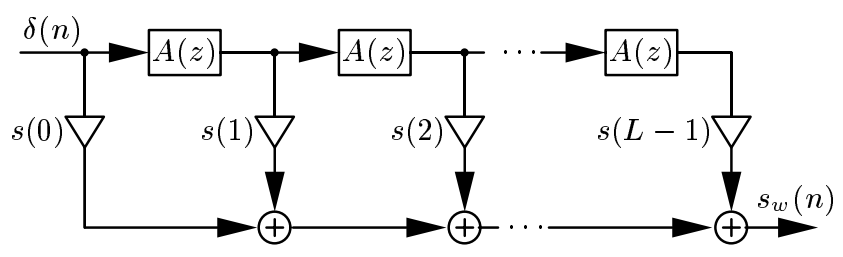

Figure 2: The warped-FIR filter structure which is used in the dewarping of the input signal and in the warping of the simulation result of the interpolated digital waveguide mesh.

\section{FREQUENCY WARPING}

The dispersion error causes frequency deviations in the digital waveguide mesh simulation results. We propose to postprocess the impulse response of the mesh using a warped-FIR filter [13, $14,15]$, which effectively introduces frequency shifting to reduce the dispersion error. The first-order allpass warping seems suitable for our problem since the frequency error curves of the interpolated mesh are monotonous and smooth (see Fig. 1(b)). Moreover, a single correction function is considered adequate since there is a small difference between maximal and minimal frequency errors. The structure used in this method is an FIR filter where every unit delay element has been replaced with a first-order allpass filter with a transfer function

$$
A(z)=\frac{z^{-1}+\lambda}{1+\lambda z^{-1}}
$$

as illustrated in Fig. 2. The extent of warping is determined by the allpass filter coefficient $\lambda$, which is the same for all the allpasses in the chain. The tap coefficients are set equal to the signal samples $s(n)$ to be warped. When a unit impulse $\delta(n)$ is fed into this filter structure, the output signal $s_{w}(n)$ is the frequency-warped version of the original signal.

\subsection{Optimization of the Warping Factor}

We have applied various different strategies in finding the optimal value for $\lambda$. In Fig. 3 we show RFE's obtained with two values of $\lambda$. In the first one (Fig. 3(a)) the maximal error was minimized. As a result the $\lambda=-0.1947$ and the error is less than $1.5 \%$ on the frequency band $\left[0,0.25 f_{s}\right]$. The disadvantage of this method is that it may produce quite large RFE already at low frequencies.

In the second optimization technique $\lambda$ is optimized such that the RFE is below a given error limit upto a maximally high frequency. In the results shown in Fig. 3(b) we have used a 1\% error limit which yields $\lambda=-0.1757$ having the RFE below $1 \%$ upto $0.220 f_{s}$.

The RFE after warping is obtained as follows:

$R F E_{\text {warped }}(\xi)=\frac{\left[k(\xi) \cdot w_{\text {ratio }}(\lambda, \xi \cdot k(\xi)) \cdot D\right]-k_{D C}}{k_{D C}} \cdot 100 \%$

where $w_{\text {ratio }}(\lambda, \xi)$ returns the ratio of warping of given frequency $\xi$, and $D=\frac{1-\lambda}{1+\lambda}$ is the phase delay at low frequencies caused by warping [11, eq. 86].

\subsection{Warped Interpolated Rectangular Mesh}

The warped interpolated rectangular mesh simulations should be conducted as follows. An arbitrary excitation signal can be used, 


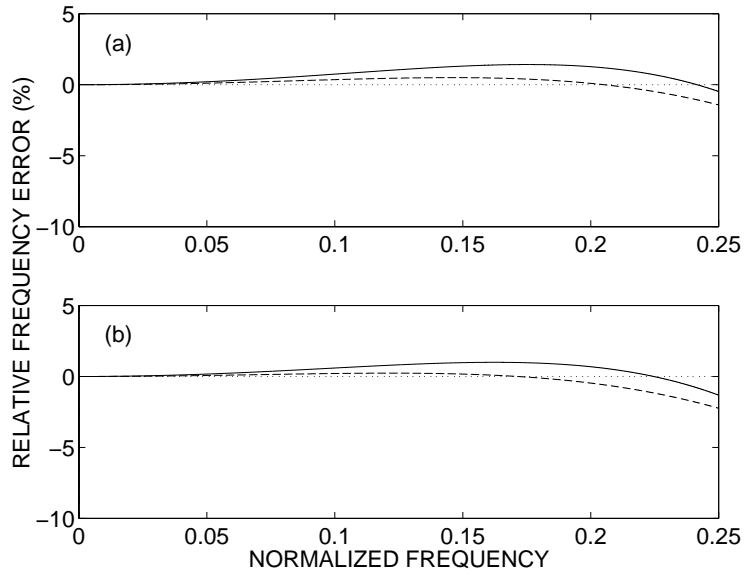

Figure 3: Relative frequency error in axial (solid) and diagonal (dashed) directions with different values of $\lambda$ such that in (a) $\lambda=-0.1947$ and in (b) $\lambda=-0.1757$ as a function of normalized temporal frequency, where 0.5 corresponds to the Nyquist frequency. The dotted line indicates the zero error.

but first it must be warped using $-\lambda$, which corresponds to dewarping, and after simulation the response should be warped using $\lambda$. The warping slows down low frequencies by $D$ and the bilinear interpolation speeds up low frequencies by $k_{D C}=1.10$. There are two ways how these effects can be taken into account: 1) by downsampling the warped output signal by factor $\frac{D}{k_{D C}}$ or 2) by increasing the spatial sampling grid size of the mesh by $\frac{D}{k_{D C}}$.

The computational cost of the proposed technique lies in the simulation itself and in warping of the excitation and the simulation result. In the original structure for each time step and for each node five summations and one binary shift is required. For the interpolated rectangular mesh the required amount of operations is nine summations and three multiplications. The warping is of computational complexity $O\left(L^{2}\right)$ where $L$ is the length of the impulse response. Thus the computational cost of warping depends only on the length of the warped signal and not on the size of the mesh.

To achieve the same accuracy as in the warped interpolated rectangular mesh, the original rectangular mesh simulations should be done with a spatial sampling grid spacing that is 2.9 times that of the original one, since the dispersion error of $1.0 \%$ is obtained at normalized temporal frequency $0.077(\approx 0.22 / 2.9)$. It means that over $8\left(\approx 2.9^{2}\right)$ times more memory is required and the number of simulation steps is nearly tripled for a given time period. Especially the memory usage may become a limiting factor.

The proposed warped interpolated rectangular mesh technique suits best for non-real-time simulations where a reasonably large mesh is used and a high frequency accuracy is required.

\section{SIMULATION EXAMPLE}

In this section, we simulate a vibrating rectangular plate using three different methods and compare the results to illustrate the effectiveness of the proposed frequency-warping technique. The ideal plate is of square shape with clamped edges. The reflec-

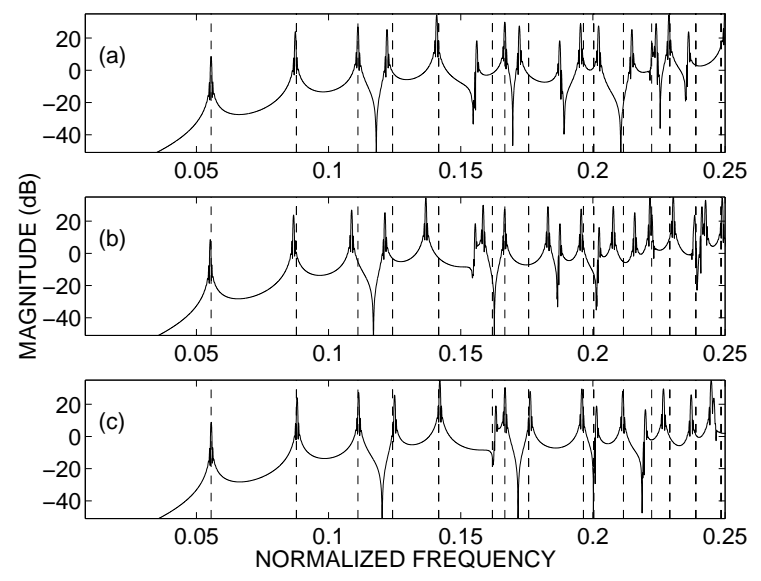

Figure 4: Eigenfrequencies of an ideal square plate, which is clamped on each boundary, simulated using (a) the original, (b) the interpolated, and (c) the warped $(\lambda=-0.1757)$ interpolated 2-D digital waveguide mesh. The ideal mode frequencies of the plate are shown with vertical dashed lines.

tions from the boundaries are implemented as reflection coefficient equal to -1 . The results in Fig. 4 are from different numerical simulations with $10 \times 10$ mesh nodes. The source is located near one corner of the plate (point $(3,3))$ and the receiver is at the opposite corner (point $(9,9)$ ). The mesh is excited with an impulse that is injected into a single node. It is also necessary to inject an attenuated and advanced impulse into its four neighboring nodes [16].

In the case of the original and the interpolated mesh, 3200 samples were computed. The coefficients of the warped-FIR filter were obtained by windowing the output signal of the interpolated mesh with the right wing of a Hanning window. The resulting warped signal was truncated to a length of 4900 samples. All the magnitude responses in Fig. 4 were obtained by windowing the signal with a right half of a Hanning window and computing a 65536-point FFT. The vertical lines in Fig. 4 show the ideal distinct eigenfrequencies of the plate which have been calculated using the Rayleigh equation [17].

Figure 4(a) presents the magnitude response obtained from the original digital waveguide mesh. All of the mode frequencies are either solved exactly or they are too low. Figure 4(b) shows the response produced with the interpolated digital waveguide mesh. It is seen that all the mode frequencies are systematically too low. In Fig. 4(c) the frequency warped $(\lambda=-0.1757)$ result is shown. Now some mode frequencies are slightly too high and some are too low. The result of the new interpolated digital waveguide mesh with frequency warping (Fig. 4(c)) is generally more accurate than those in Figs. 4(a) and 4(b). Note that in Figs. 4(b) and 4(c) instead of resampling the response, frequency has been scaled by $\frac{1}{{ }^{k_{D C}}}=$ 0.9102 and $\frac{D}{{ }^{k_{D C}}}=1.298$, respectively.

Table 1 shows the analytically solved eigenfrequencies and simulation results of the original, the interpolated, and the warped interpolated mesh. The eigenfrequencies are detected with the accuracy of the corresponding RFE curves shown in Figs. 1(a), 1(b) and 3(b) such that the error in diagonal modes $\left(n_{x}=n_{y}\right)$ is presented by dashed lines and the error in axial modes $\left(n_{x}=0 \vee n_{y}=\right.$ 0 ) by solid lines (not present in this example due to the chosen 
Table 1: Mode frequencies of the ideal square plate and the original, the interpolated, and the warped $(\lambda=-0.1757)$ interpolated mesh simulations. The error is included in parentheses.

\begin{tabular}{c|c|l|l|l}
$\begin{array}{c}\text { Mode } \\
\left(n_{x}, n_{y}\right)\end{array}$ & Ideal & Original & Interpolated & $\begin{array}{l}\text { Warped } \\
\text { Interpolated }\end{array}$ \\
\hline$(1,1)$ & .056 & $.056(0.0 \%)$ & $.055(-0.5 \%)$ & $.056(0.1 \%)$ \\
$(1,2)$ & .088 & $.087(-0.5 \%)$ & $.087(-1.2 \%)$ & $.088(0.3 \%)$ \\
$(2,2)$ & .111 & $.111(0.0 \%)$ & $.109(-2.1 \%)$ & $.111(0.2 \%)$ \\
$(1,3)$ & .124 & $.122(-1.7 \%)$ & $.121(-2.3 \%)$ & $.125(0.6 \%)$ \\
$(2,3)$ & .142 & $.141(-0.5 \%)$ & $.137(-3.4 \%)$ & $.142(0.3 \%)$ \\
$(1,4)$ & .162 & $.156(-3.6 \%)$ & $.156(-4.0 \%)$ & $.163(0.8 \%)$ \\
$(3,3)$ & .167 & $.167(0.0 \%)$ & $.158(-4.9 \%)$ & $.167(0.1 \%)$ \\
$(2,4)$ & .176 & $.172(-2.0 \%)$ & $.167(-5.2 \%)$ & $.176(0.3 \%)$ \\
$(3,4)$ & .196 & $.195(-0.6 \%)$ & $.183(-6.8 \%)$ & $.196(-0.3 \%)$ \\
$(1,5)$ & .200 & $.187(-6.4 \%)$ & $.188(-6.4 \%)$ & $.201(0.5 \%)$ \\
$(2,5)$ & .212 & $.202(-4.4 \%)$ & $.196(-7.6 \%)$ & $.211(-0.1 \%)$ \\
$(4,4)$ & .222 & $.222(0.0 \%)$ & $.202(-8.9 \%)$ & $.220(-1.1 \%)$ \\
$(3,5)$ & .229 & $.224(-2.3 \%)$ & $.208(-9.3 \%)$ & $.227(-1.0 \%)$ \\
$(1,6)$ & .239 & $.215(-10 \%)$ & $.216(-9.7 \%)$ & $.237(-0.8 \%)$ \\
$(2,6)$ & .248 & $.229(-7.9 \%)$ & $.222(-11 \%)$ & $.245(-1.5 \%)$
\end{tabular}

boundary condition). For example the error in mode $(4,4)$ is $0.0 \%$ (see Table 1) in the original mesh since there is no dispersion error in the diagonal direction (dashed line in Fig. 1(a)). The error in the non-diagonal $\left(n_{x} \neq n_{y}\right)$ modes is between the RFE of diagonal and axial directions. For example, the error of mode $(1,5)$ which should ideally be at normalized frequency 0.200 , is $0.5 \%$ too high in the warped interpolated mesh. This RFE is located between the solid and dashed lines in (Fig. 3(b)) since it is a non-diagonal mode.

\section{FUTURE WORK AND CONCLUSION}

In the future, the frequency-warping technique will be applied also to three-dimensional mesh algorithms. The interpolation technique works for rectangular 3-D meshes as shown in [12] and finding the appropriate warping factor should be straightforward. The warping technique is suitable for triangular digital waveguide mesh algorithms as well [18].

In this paper we have shown that the $1 \%$ accurate bandwidth of 2-D digital waveguide mesh simulations can be extended with the interpolated warped structure when compared with the original one. The proposed frequency warping reduces the dispersion error which is the major cause of inaccuracies in the original digital waveguide mesh.

\section{ACKNOWLEDGMENT}

The authors are grateful to Mr. Aki Härmä for helpful discussions. This work has been supported by the Academy of Finland and the Technology Development Centre, Finland (TEKES).

\section{REFERENCES}

[1] J. O. Smith, "Physical modeling using digital waveguides," Computer Music J., vol. 16, no. 4, pp. 74-87, 1992 Winter.
[2] J. O. Smith, "Principles of digital waveguide models of musical instruments," in Applications of Digital Signal Processing to Audio and Acoustics, M. Kahrs and K. Brandenburg, Eds., chapter 10, pp. 417-466. Kluwer, 1997.

[3] S. Van Duyne and J. O. Smith, "The 2-D digital waveguide mesh," in Proc. 1993 IEEE Workshop Appl. of Signal Processing to Audio and Acoustics, New Paltz, NY, Oct. 1993.

[4] S. Van Duyne and J. O. Smith, "Physical modeling with the 2-D digital waveguide mesh," in Proc. 1993 Int. Computer Music Conf., Tokyo, Japan, Sept. 1993, pp. 40-47.

[5] L. Savioja, T. Rinne, and T. Takala, "Simulation of room acoustics with a 3-D finite difference mesh," in Proc. 1994 Int. Computer Music Conf., Aarhus, Denmark, 12-17 Sept. 1994, pp. 463-466.

[6] T. Schetelig and R. Rabenstein, "Simulation of threedimensional sound propagation with multidimensional wave digital filters," in Proc. IEEE Int. Conf. Acoust., Speech, Signal Processing, Seattle, Washington, 12-16 May 1998, vol. 6, pp. 3537-3540.

[7] L. Savioja and V. Välimäki, "Improved discrete-time modeling of multi-dimensional wave propagation using the interpolated digital waveguide mesh," in Proc. IEEE Int. Conf. Acoust., Speech, Signal Processing, Munich, Germany, 1924 April 1997, vol. 1, pp. 459-462.

[8] S. Van Duyne and J. O. Smith, "The tetrahedral digital waveguide mesh," in Proc. 1995 IEEE Workshop on Applications of Signal Processing to Audio and Acoustics, New Paltz, NY, USA, Oct. 1995.

[9] S. Van Duyne and J. O. Smith, "The 3D tetrahedral digital waveguide mesh with musical applications," in Proc. 1996 Int. Computer Music Conf., Hong Kong, 19-24 Aug. 1996, pp. 9-16.

[10] F. Fontana and D. Rocchesso, "Physical modeling of membranes for percussion instruments," Acustica, vol. 84, no. 3, pp. 529-542, May/June 1998.

[11] T. I. Laakso, V. Välimäki, M. Karjalainen, and U. K. Laine, "Splitting the unit delay - tools for fractional delay filter design," IEEE Signal Processing Mag., vol. 13, no. 1, pp. 3060, Jan. 1996.

[12] L. Savioja, "Improving the three-dimensional digital waveguide mesh by interpolation," in Proc. Nordic Acoustical Meeting (NAM'98), Stockholm, Sweden, 7-9 Sept 1998.

[13] A. Oppenheim, D. Johnson, and K. Steiglitz, "Computation of spectra with unequal resolution using the Fast Fourier Transform," Proc. IEEE, vol. 59, no. 2, pp. 299-301, Feb. 1971.

[14] U. K. Laine, M. Karjalainen, and T. Altosaar, "Warped linear prediction (WLP) in speech and audio processing," in Proc. IEEE Int. Conf. Acoust., Speech, Signal Processing, Adelaide, Australia, 19-22 April 1994, vol. 3, pp. 349-352.

[15] M. Karjalainen and J. O. Smith, "Body modeling techniques for string instrument synthesis," in Proc. 1996 Int. Computer Music Conf., Hong Kong, 19-24 Aug. 1996, pp. 232-239.

[16] L. Savioja, M. Karjalainen, and T. Takala, "DSP formulation of a finite difference method for room acoustics simulation," in Proc. 1996 IEEE Nordic Signal Processing Symp., Espoo, Finland, 24-27 Sept. 1996, pp. 455-458.

[17] J. W. S. Rayleigh, The Theory of Sound, vol. 2, Macmillan Company, 1896, Reprinted by Dover, New York, 1945, pp. 70-71.

[18] L. Savioja and V. Välimäki, "Reduction of the dispersion error in the triangular digital waveguide mesh using frequency warping," Unpublished manuscript, August 1998. 